FACTA UNIVERSITATIS (NIŠ)

Ser. Math. Inform. Vol. 35, No 4 (2020), 929-937

https://doi.org/10.22190/FUMI2004929B

\title{
CHARACTERIZATION OF SOME BIDERIVATIONS ON TRIANGULAR BANACH ALGEBRAS
}

\author{
Sedigheh Barootkoob
}

(C) 2020 by University of Niš, Serbia | Creative Commons Licence: CC BY-NC-ND

\begin{abstract}
Let $A$ and $B$ be unital Banach algebras, $X$ be a unital $A$-B-module and $T$ be the triangular Banach algebra associated to $A, B$ and $X$. The structure of some derivations on triangular Banach algebras was studied by some authors. Note that despite the apparent similarity between derivations and biderivations and also inner derivations and inner biderivations, there are fundamental differences between them. Although there are some studies of biderivations on triangular Banach algebras, any of them do not completely determine the structure of biderivations on triangular Banach algebras. In this paper, we completely characterize biderivations and inner biderivations from $T \times T$ to $T^{*}$ and we show that the first bicohomology group $B H^{1}\left(T, T^{*}\right)$ is equal to $B H^{1}\left(A, A^{*}\right) \oplus B H^{1}\left(B, B^{*}\right)$.
\end{abstract}

Keywords: unital Banach algebras; triangular Banach algebra; bicohomology group; biderivations.

\section{Introduction}

A derivation from a Banach algebra $A$ to a Banach $A$-module $X$ is a bounded linear mapping $d: A \rightarrow X$ such that for each $a, b \in A, d(a b)=d(a) b+a d(b)$. For each $x \in X$ the mapping $\delta_{x}: a \rightarrow a x-x a,(a \in A)$ is a bounded derivation, called an inner derivation.

Let $A$ be a Banach algebra and $X$ be an $A$-module. A bounded bilinear mapping $D: A \times A \rightarrow X$ is called a biderivation if $D$ is a derivation with respect to both arguments. That is, the mappings ${ }_{a} D: A \rightarrow X$ and $D_{b}: A \rightarrow X$ where

$$
{ }_{a} D(b)=D(a, b)=D_{b}(a) \quad(a, b \in A),
$$

are derivations. We denote the space of such biderivations by $B Z^{1}(A, X)$.

Received October 17, 2019; accepted November 09, 2020

2020 Mathematics Subject Classification. Primary 15A78; Secondary 46H25 
Let $x \in Z(A, X)=\{x \in X ; a x=x a \quad \forall a \in A\}$. The map $D_{x}: A \times A \rightarrow X$ that

$$
D_{x}(a, b)=x[a, b]=x a b-x b a \quad(a, b \in A),
$$

is a basic example of a biderivation which is called an inner biderivation. We denote the space of such inner biderivations by $B N^{1}(A, X)$. Also we define the first bicohomology group $B H^{1}(A, X)$ as follows,

$$
B H^{1}(A, X)=\frac{B Z^{1}(A, X)}{B N^{1}(A, X)} .
$$

For more applications and details about biderivations see [6, Section 3]. Also see $[5,8]$, in which the structures of some biderivations on triangular algebras and generalized matrix algebras and when these biderivations on these algebras are inner, were studied.

Let $A$ and $B$ be Banach algebras and $X$ be an $A$ - $B$-module. Then the algebra

$$
T=\left\{\left(\begin{array}{cc}
a & x \\
0 & b
\end{array}\right) ; a \in A, x \in X, b \in B\right\}
$$

equipped with the usual addition and multiplication of matrix and with the norm

$$
\left\|\left(\begin{array}{cc}
a & x \\
0 & b
\end{array}\right)\right\|=\|a\|+\|x\|+\|b\|
$$

is a Banach algebra which is called triangular Banach algebra associated to $X$. Then the dual of triangular Banach algebra $T$ is

$$
T^{*}=\left\{\left(\begin{array}{cc}
f & h \\
0 & g
\end{array}\right) ; f \in A^{*}, h \in X^{*}, g \in B^{*}\right\}
$$

where $\left(\begin{array}{ll}f & h \\ 0 & g\end{array}\right)\left(\left(\begin{array}{ll}a & x \\ 0 & b\end{array}\right)\right)=f(a)+h(x)+g(b)$.

Recall that for every Banach $A$-module $X$ the dual space $X^{*}$ is a Banach $A$ module with module structures $a \cdot f$ and $f \cdot a$ that $a \cdot f(x)=f(x a)$ and $f \cdot a(x)=$ $f(a x)$. So $T^{*}$ is a $T$-module with the module actions

$$
\left(\begin{array}{ll}
a & x \\
0 & b
\end{array}\right) \cdot\left(\begin{array}{ll}
f & h \\
0 & g
\end{array}\right)=\left(\begin{array}{cc}
a \cdot f+x \cdot h & b \cdot h \\
0 & b \cdot g
\end{array}\right)
$$

and

$$
\left(\begin{array}{ll}
f & h \\
0 & g
\end{array}\right) \cdot\left(\begin{array}{ll}
a & x \\
0 & b
\end{array}\right)=\left(\begin{array}{cc}
f \cdot a & h \cdot a \\
0 & h \cdot x+g \cdot b
\end{array}\right)
$$

for every $\left(\begin{array}{cc}a & x \\ 0 & b\end{array}\right) \in T$ and $\left(\begin{array}{cc}f & h \\ 0 & g\end{array}\right) \in T^{*}$.

A Banach algebra $A$ is called weakly amenable if every derivation from $A$ to $A^{*}$ is an inner derivation. The concept of weak amenability of Banach algebras was 
introduced by Bade, Curtis and Dales [1] for commutative Banach algebras and then by Johnson [10] for a general Banach algebra.

In this paper, we consider $A$ and $B$ as unital Banach algebras and $X$ as a unital $A$-B-module, that is, $1_{A} x=x 1_{B}=x$, for every $x \in X$. We characterize the biderivations from $T \times T$ to $T^{*}$. In particular, we show that $B H^{1}\left(T, T^{*}\right)=$ $B H^{1}\left(A, A^{*}\right) \oplus B H^{1}\left(B, B^{*}\right)$.

\section{Biderivations and biamenability of triangular Banach algebras}

Similar to the definitions of amenability or weak amenability of Banach algebras we may define the notions of biamenability [2] or weak biamenability of Banach algebras [3].

Definition 2.1. We say that a Banach algebra $A$ is weakly biamenable if

$$
B H^{1}\left(A, A^{*}\right)=\{0\}
$$

Example 2.1. (i) Let $\mathfrak{A}$ be a Banach space and $\theta \in \mathfrak{A}^{*}$. Then $\mathfrak{A}$ with the product

$$
a b=\theta(a) b \quad(a, b \in \mathfrak{A}),
$$

is a Banach algebra and $\theta$ becomes a homomorphism. Also for each $h \in \mathfrak{A}^{*}$ and $a, b, c \in \mathfrak{A}$ we have $h \cdot a=\theta(a) h$ and $a \cdot h=h(a) \theta$ and since $\theta(a b)=\theta(b a)$, we have $[a, b] c=[b, a] c$. Now consider $f \in \mathfrak{A}^{*}$ such that for some $a_{0}, b_{0} \in \mathfrak{A}, \theta\left(a_{0}\right) f\left(b_{0}\right) \neq$ $f\left(a_{0}\right) \theta\left(b_{0}\right)$. Define the biderivation $D: \mathfrak{A} \times \mathfrak{A} \rightarrow A^{*}$ by $D(a, b)=\delta_{\delta_{f}(a)}(b)$, for each $a, b \in \mathfrak{A}$. Then since $D$ is non zero and the only inner biderivation from $\mathfrak{A} \times \mathfrak{A}$ into $\mathfrak{A}^{*}$ is zero, we conclude that $\mathfrak{A}$ with this product is not weakly biamenable.

(ii) Let $B(H)$ be the Banach algebra of operators on Hilbert space $H$ and $D: B(H) \times$ $B(H) \rightarrow B(H)^{*}$ be a biderivation. Then similar to Lemma 1 of $[5] D(T, S)[U, V]=$ $[T, S] D(U, V)$ for each $T, S, U, V \in B(H)$. Also by Lemma 5.8 of [12] $B(H)=$ $\operatorname{span}\{U V-V U ; V, U \in B(H)\}$. Therefore there exist $\left\{U_{i}\right\},\left\{V_{i}\right\}$ in $B(H)$ such that $I=\sum_{i}\left[U_{i}, V i\right]$. Now we have

$$
\begin{aligned}
D(T, S) & =D(T, S) I \\
& =D(T, S) \sum_{i}\left[U_{i}, V i\right] \\
& =\sum_{i}[T, S] D\left(U_{i}, V_{i}\right) \\
& =[T, S] \sum_{i} D\left(U_{i}, V_{i}\right)
\end{aligned}
$$

and similarly $D(T, S)=\sum_{i} D\left(U_{i}, V_{i}\right)[T, S]$. So if we put $x=\sum_{i} D\left(U_{i}, V_{i}\right)$, then $x \in Z\left(B(H), B(H)^{*}\right)$ and $D(T, S)=x[T, S]$. That is $D$ is an inner biderivation and so $B(H)$ is weakly biamenable.

Note that despite the apparent similarity between derivations and biderivations and also inner derivations and inner biderivations, there are fundamental differences between them. Especially when a biderivation wants to be an inner bidetivation these differences become more apparent. A part of these differences comes from the nature of biderivations which depend on two components. Another essential part 
of these differences goes back to the definition of inner biderivations which depends on the implemented elements that should be in $Z(A, X)$. According to this, the concept of amenability and also weak amenability have a different nature from biamenability and weak biamenability, respectively. Indeed, there are examples of Banach algebras that are biamenable while they are not amenable and there are Banach algebras that are amenable while they are not biamenable [2]. Also, if we consider the definition of a biamenable group $G$ such that $G \times G$ is amenable, then we see that the Johnson's theorem [11] is not valid for biamenability. Indeed, each abelian group $G$ is biamenable while the commutative group algebra $L^{1}(G)$ is not biamenable [2]. Of course, the situation of weak biamenability is better than biamenability, and many similar results of [4] and [7] are valid for weak biamenability of Banach algebras. Also, for each locally compact abelian group $G, L^{1}(G)$ is weakly biamenable. For more detales, see [3].

The next theorem characterizes all biderivations from $T \times T$ to $T^{*}$.

Theorem 2.1. A bilinear mapping $D: T \times T \rightarrow T^{*}$ is a biderivation if and only if there exist biderivations $d_{A}: A \times A \rightarrow A^{*}$ and $d_{B}: B \times B \rightarrow B^{*}$ such that

$$
D\left(\left(\begin{array}{ll}
a & x \\
0 & b
\end{array}\right),\left(\begin{array}{cc}
a^{\prime} & x^{\prime} \\
0 & b^{\prime}
\end{array}\right)\right)=\left(\begin{array}{cc}
d_{A}\left(a, a^{\prime}\right) & 0 \\
0 & d_{B}\left(b, b^{\prime}\right)
\end{array}\right) .
$$

Proof. It is easy to verify that if

$$
D\left(\left(\begin{array}{ll}
a & x \\
0 & b
\end{array}\right),\left(\begin{array}{cc}
a^{\prime} & x^{\prime} \\
0 & b^{\prime}
\end{array}\right)\right)=\left(\begin{array}{cc}
d_{A}\left(a, a^{\prime}\right) & 0 \\
0 & d_{B}\left(b, b^{\prime}\right)
\end{array}\right),
$$

for some biderivations $d_{A}: A \times A \rightarrow A^{*}$ and $d_{B}: B \times B \rightarrow B^{*}$, then $D$ is a biderivation.

Conversely, let $D: T \times T \rightarrow T^{*}$ be a biderivation. Since for every $a, a^{\prime} \in$ $A, b, b^{\prime} \in B$ and $x, x^{\prime} \in X$ we have

$$
\begin{aligned}
& \left(\begin{array}{ll}
a & x \\
0 & b
\end{array}\right)^{D}\left(\left(\begin{array}{cc}
a^{\prime} & x^{\prime} \\
0 & b^{\prime}
\end{array}\right)\right)=D\left(\left(\begin{array}{cc}
a & x \\
0 & b
\end{array}\right),\left(\begin{array}{cc}
a^{\prime} & x^{\prime} \\
0 & b^{\prime}
\end{array}\right)\right)= \\
& D\left(\begin{array}{ll}
a^{\prime} & x^{\prime} \\
0 & b^{\prime}
\end{array}\right)\left(\left(\begin{array}{ll}
a & x \\
0 & b
\end{array}\right)\right)
\end{aligned}
$$

and $\left(\begin{array}{cc}a & x \\ 0 & b\end{array}\right)^{D \text { and } D}\left(\begin{array}{cc}a^{\prime} & x^{\prime} \\ 0 & b^{\prime}\end{array}\right)$ are derivations, according to [9] there exist derivations $d_{\left(a^{\prime}, x^{\prime}, b^{\prime}\right)}, d_{(a, x, b)}^{\prime}: A \rightarrow A^{*}$ and $\delta_{\left(a^{\prime}, x^{\prime}, b^{\prime}\right)}, \delta_{(a, x, b)}^{\prime}: B \rightarrow B^{*}$ and also 
$k_{\left(a^{\prime}, x^{\prime}, b^{\prime}\right)}, k_{(a, x, b)}^{\prime} \in X^{*}$ such that

$$
\begin{aligned}
& \left(\begin{array}{cc}
d_{\left(a^{\prime}, x^{\prime}, b^{\prime}\right)}(a)-x k_{\left(a^{\prime}, x^{\prime}, b^{\prime}\right)} & k_{\left(a^{\prime}, x^{\prime}, b^{\prime}\right)} a-b k_{\left(a^{\prime}, x^{\prime}, b^{\prime}\right)} \\
0 & k_{\left(a^{\prime}, x^{\prime}, b^{\prime}\right)} x+\delta_{\left(a^{\prime}, x^{\prime}, b^{\prime}\right)}(b)
\end{array}\right) \\
= & D\left(\begin{array}{cc}
a^{\prime} & x^{\prime} \\
0 & b^{\prime}
\end{array}\right)\left(\left(\begin{array}{cc}
a & x \\
0 & b
\end{array}\right)\right) \\
= & D\left(\left(\begin{array}{cc}
a & x \\
0 & b
\end{array}\right),\left(\begin{array}{cc}
a^{\prime} & x^{\prime} \\
0 & b^{\prime}
\end{array}\right)\right) \\
= & \left.\left.\left(\begin{array}{cc}
a & x \\
0 & b
\end{array}\right) \begin{array}{cc}
a^{\prime} & x^{\prime} \\
0 & b^{\prime}
\end{array}\right)\right) \\
= & \left(\begin{array}{cc}
d_{(a, x, b)}^{\prime}\left(a^{\prime}\right)-x^{\prime} k_{(a, x, b)}^{\prime} & k_{(a, x, b)}^{\prime} a^{\prime}-b^{\prime} k_{(a, x, b)}^{\prime} \\
0 & k_{(a, x, b)}^{\prime} x^{\prime}+\delta_{(a, x, b)}^{\prime}\left(b^{\prime}\right)
\end{array}\right) .
\end{aligned}
$$

In particular

$$
\begin{aligned}
\left(\begin{array}{cc}
d_{\left(a^{\prime}, 0,0\right)}(a) & k_{\left(a^{\prime}, 0,0\right)} a \\
0 & 0
\end{array}\right) & =D\left(\left(\begin{array}{cc}
a & 0 \\
0 & 0
\end{array}\right),\left(\begin{array}{cc}
a^{\prime} & 0 \\
0 & 0
\end{array}\right)\right) \\
& =\left(\begin{array}{cc}
d_{(a, 0,0)}^{\prime}\left(a^{\prime}\right) & k_{(a, 0,0)}^{\prime} a^{\prime} \\
0 & 0
\end{array}\right) .
\end{aligned}
$$

Define $d_{A}: A \times A \rightarrow A^{*}$ by $d_{A}\left(a, a^{\prime}\right)=d_{\left(a^{\prime}, 0,0\right)}(a)=d_{(a, 0,0)}^{\prime}\left(a^{\prime}\right)$. Then obviously $d_{A}$ is a bounded biderivation.

Similarly we can define the biderivation $d_{B}: B \times B \rightarrow B^{*}$ such that $d_{B}\left(b, b^{\prime}\right)=$ $\delta_{\left(0,0, b^{\prime}\right)}(b)=\delta_{(0,0, b)}^{\prime}\left(b^{\prime}\right)$. Also we have

$$
\begin{aligned}
\left(\begin{array}{cc}
d_{\left(0,0, b^{\prime}\right)}(a) & k_{\left(0,0, b^{\prime}\right)} a \\
0 & 0
\end{array}\right) & =D\left(\left(\begin{array}{cc}
a & 0 \\
0 & 0
\end{array}\right),\left(\begin{array}{cc}
0 & 0 \\
0 & b^{\prime}
\end{array}\right)\right) \\
& =\left(\begin{array}{cc}
0 & -b^{\prime} k_{(a, 0,0)}^{\prime} \\
0 & \delta_{(a, 0,0)}^{\prime}\left(b^{\prime}\right)
\end{array}\right) .
\end{aligned}
$$

So

$$
d_{\left(0,0, b^{\prime}\right)}(a)=0, \quad \delta_{(a, 0,0)}^{\prime}\left(b^{\prime}\right)=0 \quad \text { and } \quad k_{\left(0,0, b^{\prime}\right)} a=-b^{\prime} k_{(a, 0,0)}^{\prime} .
$$

On the other hand

$$
\begin{aligned}
\left(\begin{array}{cc}
d_{\left(0, x^{\prime}, 0\right)}(a) & k_{\left(0, x^{\prime}, 0\right)} a \\
0 & 0
\end{array}\right) & =D\left(\left(\begin{array}{ll}
a & 0 \\
0 & 0
\end{array}\right),\left(\begin{array}{cc}
0 & x^{\prime} \\
0 & 0
\end{array}\right)\right) \\
& =\left(\begin{array}{cc}
-x^{\prime} k_{(a, 0,0)}^{\prime} & 0 \\
0 & k_{(a, 0,0)}^{\prime} x^{\prime}
\end{array}\right) .
\end{aligned}
$$

Therefore $d_{\left(0, x^{\prime}, 0\right)}(a)=-x^{\prime} k_{(a, 0,0)}^{\prime}, k_{\left(a^{\prime}, 0,0\right)} a=0$ and $k_{(a, 0,0)}^{\prime} x^{\prime}=0$. In particular for each $x^{\prime} \in X,-x^{\prime} k_{\left(1_{A}, 0,0\right)}^{\prime}=d_{\left(0, x^{\prime}, 0\right)}\left(1_{A}\right)=0$ and since $X$ is a unital $A$ module, $k_{\left(1_{A}, 0,0\right)}^{\prime}=0$. On the other hand by $(2.1)$ we have $k_{\left(0,0,1_{B}\right)}=k_{\left(0,0,1_{B}\right)} 1_{A}=$ 
$-1_{B} k_{\left(1_{A}, 0,0\right)}^{\prime}=0$ and hence

$$
d_{\left(0, x^{\prime}, 0\right)}(a)=-x^{\prime} k_{(a, 0,0)}^{\prime}=-x^{\prime} 1_{B} k_{(a, 0,0)}^{\prime}=x^{\prime} k_{\left(0,0,1_{B}\right)} a=0 .
$$

So

$$
\begin{aligned}
D\left(\left(\begin{array}{ll}
a & 0 \\
0 & 0
\end{array}\right),\left(\begin{array}{cc}
a^{\prime} & x^{\prime} \\
0 & b^{\prime}
\end{array}\right)\right) & =D\left(\left(\begin{array}{ll}
a & 0 \\
0 & 0
\end{array}\right),\left(\begin{array}{cc}
a^{\prime} & 0 \\
0 & 0
\end{array}\right)\right) \\
& +D\left(\left(\begin{array}{ll}
a & 0 \\
0 & 0
\end{array}\right),\left(\begin{array}{cc}
0 & x^{\prime} \\
0 & 0
\end{array}\right)\right) \\
& +D\left(\left(\begin{array}{ll}
a & 0 \\
0 & 0
\end{array}\right),\left(\begin{array}{cc}
0 & 0 \\
0 & b^{\prime}
\end{array}\right)\right) \\
& =\left(\begin{array}{cc}
d_{A}\left(a, a^{\prime}\right) & k_{\left(a^{\prime}, 0,0\right)} a+k_{\left(0, x^{\prime}, 0\right)} a+k_{\left(0,0, b^{\prime}\right)} a \\
0 & 0
\end{array}\right) .
\end{aligned}
$$

Now since $k_{\left(a+a^{\prime}, 0,0\right)}=k_{(a, 0,0)}+k_{\left(a^{\prime}, 0,0\right)}, k_{\left(0, x+x^{\prime}, 0\right)}=k_{(0, x, 0)}+k_{\left(0, x^{\prime}, 0\right)}$ and $k_{\left(0,0, b+b^{\prime}\right)}=$ $k_{(0,0, b)}+k_{\left(0,0, b^{\prime}\right)}$, we can define the linear mapping

$$
\begin{aligned}
h: A \oplus X \oplus B & \rightarrow X^{*} \\
(a, x, b) & \mapsto k_{(a, 0,0)}+k_{(0, x, 0)}+k_{(0,0, b)}
\end{aligned}
$$

and we have $D\left(\left(\begin{array}{cc}a & 0 \\ 0 & 0\end{array}\right),\left(\begin{array}{cc}a^{\prime} & x^{\prime} \\ 0 & b^{\prime}\end{array}\right)\right)=\left(\begin{array}{cc}d_{A}\left(a, a^{\prime}\right) & h\left(a^{\prime}, x^{\prime}, b^{\prime}\right) a \\ 0 & 0\end{array}\right)$.

Similarly we have

$$
D\left(\left(\begin{array}{ll}
0 & 0 \\
0 & b
\end{array}\right),\left(\begin{array}{cc}
a^{\prime} & x^{\prime} \\
0 & b^{\prime}
\end{array}\right)\right)=\left(\begin{array}{cc}
0 & -b h\left(a^{\prime}, x^{\prime}, b^{\prime}\right) \\
0 & d_{B}\left(b, b^{\prime}\right)
\end{array}\right)
$$

and

$$
D\left(\left(\begin{array}{ll}
0 & x \\
0 & 0
\end{array}\right),\left(\begin{array}{cc}
a^{\prime} & x^{\prime} \\
0 & b^{\prime}
\end{array}\right)\right)=\left(\begin{array}{cc}
-x h\left(a^{\prime}, x^{\prime}, b^{\prime}\right) & 0 \\
0 & h\left(a^{\prime}, x^{\prime}, b^{\prime}\right) x
\end{array}\right) .
$$

So we have

$$
\begin{aligned}
& D\left(\left(\begin{array}{ll}
a & x \\
0 & b
\end{array}\right),\left(\begin{array}{cc}
a^{\prime} & x^{\prime} \\
0 & b^{\prime}
\end{array}\right)\right) \\
= & \left(\begin{array}{cc}
d_{A}\left(a, a^{\prime}\right)-x h\left(a^{\prime}, x^{\prime}, b^{\prime}\right) & h\left(a^{\prime}, x^{\prime}, b^{\prime}\right) a-b h\left(a^{\prime}, x^{\prime}, b^{\prime}\right) \\
0 & h\left(a^{\prime}, x^{\prime}, b^{\prime}\right) x+d_{B}\left(b, b^{\prime}\right)
\end{array}\right) .
\end{aligned}
$$

Also we can show similarly there is a bounded linear mappings $h^{\prime}: A \oplus X \oplus B \rightarrow X^{*}$ such that

$$
\begin{aligned}
& D\left(\left(\begin{array}{ll}
a & x \\
0 & b
\end{array}\right),\left(\begin{array}{cc}
a^{\prime} & x^{\prime} \\
0 & b^{\prime}
\end{array}\right)\right) \\
= & \left(\begin{array}{cc}
d_{A}\left(a, a^{\prime}\right)-x^{\prime} h^{\prime}(a, x, b) & h^{\prime}(a, x, b) a^{\prime}-b^{\prime} h^{\prime}(a, x, b) \\
0 & h^{\prime}(a, x, b) x^{\prime}+d_{B}\left(b, b^{\prime}\right)
\end{array}\right) .
\end{aligned}
$$


Therefore $h\left(a^{\prime}, x^{\prime}, b^{\prime}\right) a-b h\left(a^{\prime}, b^{\prime}, x^{\prime}\right)=h^{\prime}(a, x, b) a^{\prime}-b^{\prime} h^{\prime}(a, x, b)$. So

$$
\begin{aligned}
h(a, x, b) & =h(a, x, b) 1_{A}-0_{B} h(a, x, b) \\
& =h^{\prime}\left(1_{A} \cdot 0,0\right) a-b h^{\prime}\left(1_{A}, 0,0\right) \\
& =k^{\prime}\left(1_{A}, 0,0\right) a-b k^{\prime}\left(1_{A}, 0,0\right) \\
& =0 .
\end{aligned}
$$

That is,

$$
D\left(\left(\begin{array}{ll}
a & x \\
0 & b
\end{array}\right),\left(\begin{array}{cc}
a^{\prime} & x^{\prime} \\
0 & b^{\prime}
\end{array}\right)\right)=\left(\begin{array}{cc}
d_{A}\left(a, a^{\prime}\right) & 0 \\
0 & d_{B}\left(b, b^{\prime}\right)
\end{array}\right) .
$$

Proposition 2.1. The biderivation $D: T \times T \rightarrow T^{*}$ which is defined for each $a, a^{\prime} \in A, b, b^{\prime} \in B$ and $x, x^{\prime} \in X$, by

$$
D\left(\left(\begin{array}{ll}
a & x \\
0 & b
\end{array}\right),\left(\begin{array}{cc}
a^{\prime} & x^{\prime} \\
0 & b^{\prime}
\end{array}\right)\right)=\left(\begin{array}{cc}
d_{A}\left(a, a^{\prime}\right) & 0 \\
0 & d_{B}\left(b, b^{\prime}\right)
\end{array}\right)
$$

is an inner biderivation if and only if $d_{A}$ and $d_{B}$ are inner biderivations.

Proof. If $d_{A}$ and $d_{B}$ are inner biderivations, then there are $f \in Z\left(A, A^{*}\right)$ and $g \in Z\left(B, B^{*}\right)$ such that for each $a, a^{\prime} \in A, d_{A}\left(a, a^{\prime}\right)=f\left[a, a^{\prime}\right]=f a a^{\prime}-f a^{\prime} a$ and for each $b, b^{\prime} \in B, d_{B}\left(b, b^{\prime}\right)=g\left[b, b^{\prime}\right]=g b b^{\prime}-g b^{\prime} b$. Now we have

$$
\begin{aligned}
D\left(\left(\begin{array}{ll}
a & x \\
0 & b
\end{array}\right),\left(\begin{array}{cc}
a^{\prime} & x^{\prime} \\
0 & b^{\prime}
\end{array}\right)\right) & =\left(\begin{array}{cc}
d_{A}\left(a, a^{\prime}\right) & 0 \\
0 & d_{B}\left(b, b^{\prime}\right)
\end{array}\right) \\
& =\left(\begin{array}{cc}
f\left[a, a^{\prime}\right] & 0 \\
0 & g\left[b, b^{\prime}\right]
\end{array}\right) \\
& =\left(\begin{array}{cc}
f & 0 \\
0 & g
\end{array}\right)\left[\left(\begin{array}{ll}
a & x \\
0 & b
\end{array}\right),\left(\begin{array}{cc}
a^{\prime} & x^{\prime} \\
0 & b^{\prime}
\end{array}\right)\right] .
\end{aligned}
$$

Also it is easy to see that $\left(\begin{array}{ll}f & 0 \\ 0 & g\end{array}\right) \in Z\left(T, T^{*}\right)$ if and only if $f \in Z\left(A, A^{*}\right)$ and $g \in Z\left(B, B^{*}\right)$. Hence $D$ is an inner biderivation.

Conversely, if $D$ is an inner biderivation, then there exists $\left(\begin{array}{cc}f & h \\ 0 & g\end{array}\right) \in Z\left(T, T^{*}\right)$ such that

$$
D\left(\left(\begin{array}{ll}
a & x \\
0 & b
\end{array}\right),\left(\begin{array}{cc}
a^{\prime} & x^{\prime} \\
0 & b^{\prime}
\end{array}\right)\right)=\left(\begin{array}{cc}
f & h \\
0 & g
\end{array}\right)\left[\left(\begin{array}{cc}
a & x \\
0 & b
\end{array}\right),\left(\begin{array}{cc}
a^{\prime} & x^{\prime} \\
0 & b^{\prime}
\end{array}\right)\right] .
$$

In particular

$$
\begin{aligned}
\left(\begin{array}{cc}
d_{A}\left(a, a^{\prime}\right) & 0 \\
0 & 0
\end{array}\right) & =D\left(\left(\begin{array}{ll}
a & 0 \\
0 & 0
\end{array}\right),\left(\begin{array}{ll}
a^{\prime} & 0 \\
0 & 0
\end{array}\right)\right) \\
& =\left(\begin{array}{cc}
f & h \\
0 & g
\end{array}\right)\left[\left(\begin{array}{cc}
a & 0 \\
0 & 0
\end{array}\right),\left(\begin{array}{cc}
a^{\prime} & 0 \\
0 & 0
\end{array}\right)\right] \\
& =\left(\begin{array}{cc}
f\left[a, a^{\prime}\right] & h\left[a, a^{\prime}\right] \\
0 & 0
\end{array}\right) .
\end{aligned}
$$


Hence $d_{A}\left(a, a^{\prime}\right)=f\left[a, a^{\prime}\right]$ and $h\left[a, a^{\prime}\right]=0$. On the other hand for each $a \in A$ we have

$$
\begin{aligned}
\left(\begin{array}{cc}
f a & h a \\
0 & 0
\end{array}\right) & =\left(\begin{array}{cc}
f & h \\
0 & g
\end{array}\right)\left(\begin{array}{ll}
a & 0 \\
0 & 0 \\
a & 0 \\
0 & 0
\end{array}\right)\left(\begin{array}{cc}
f & h \\
0 & g
\end{array}\right) \\
& =\left(\begin{array}{cc}
a f & 0 \\
0 & 0
\end{array}\right) .
\end{aligned}
$$

That is $f \in Z\left(A, A^{*}\right)$ and $h a=0$, that is $h=0$. So $d_{A}$ is an inner biderivation. Similarly we can show that $d_{B}$ is an inner biderivation.

Note that in the latter proposition it is also proved that

$$
Z\left(T, T^{*}\right)=\left\{\left(\begin{array}{cc}
f & 0 \\
0 & g
\end{array}\right) ; f \in Z\left(A, A^{*}\right), g \in Z\left(B, B^{*}\right)\right\}
$$

Theorem 2.2. $B H^{1}\left(T \times T, T^{*}\right)=B H^{1}\left(A \times A, A^{*}\right) \oplus B H^{1}\left(B \times B, B^{*}\right)$

Proof. Define

$$
\begin{aligned}
\varphi: B Z^{1}\left(A \times A, A^{*}\right) \oplus B Z^{1}\left(B \times B, B^{*}\right) & \rightarrow B H^{1}\left(T \times T, T^{*}\right), \\
\left(d_{A}, d_{B}\right) & \mapsto\left[\left(\begin{array}{cc}
d_{A} & 0 \\
0 & d_{B}
\end{array}\right)\right]
\end{aligned}
$$

where $\left[\left(\begin{array}{cc}d_{A} & 0 \\ 0 & d_{B}\end{array}\right)\right]$ is the equivalent class of $\left(\begin{array}{cc}d_{A} & 0 \\ 0 & d_{B}\end{array}\right)$ in $B H^{1}\left(T \times T, T^{*}\right)$.

Then by Theorem 2.1, $\varphi$ is onto and by Proposition 2.1 we have

$$
\begin{aligned}
\operatorname{ker} \varphi & =\left\{\left(d_{A}, d_{B}\right) ;\left(\begin{array}{cc}
d_{A} & 0 \\
0 & d_{B}
\end{array}\right) \text { is inner }\right\} \\
& =\left\{\left(d_{A}, d_{B}\right) ; d_{A} \text { and } d_{B} \text { are inner }\right\} \\
& =B N^{1}\left(A \times A, A^{*}\right) \oplus B N^{1}\left(B \times B, B^{*}\right)
\end{aligned}
$$

Therefore

$$
\begin{aligned}
B H^{1}\left(T \times T, T^{*}\right) & =\frac{B Z^{1}\left(A \times A, A^{*}\right) \oplus B Z^{1}\left(B \times B, B^{*}\right)}{B N^{1}\left(A \times A, A^{*}\right) \oplus B N^{1}\left(B \times B, B^{*}\right)} \\
& =B H^{1}\left(A \times A, A^{*}\right) \oplus B H^{1}\left(B \times B, B^{*}\right)
\end{aligned}
$$

Corollary 2.1. $T$ is weakly biamenable if and only if $A$ and $B$ are weakly biamenable. 
For example if $A$ is a commutative Banach algebra and there is a non zero biderivation from $A \times A$ into $A^{*}$, then since the only inner biderivation from $A \times A$ into $A^{*}$ is zero, $A$ and therefore $T$ are not weakly biamenable.

Acknowledgment. The useful comments of the anonymous referees are gratefully acknowledged.

\section{REF E R E N C E S}

1. W. G. Bade, P. C. Curtis, and H. G. Dales, Amenability and weak amenability for Beurling and Lipschitz algebras. Proc. London Math. Soc. 55 (1987), 359-377.

2. S. BАRооткоов, On biamenability of Banach algebras. preprint.

3. S. Bавооткоов, On n-weak biamenability of Banach algebras. preprint.

4. S. Barootkoob and H.R. Ebrahimi Vishki, Lifting derivations and $n$-weak amenability of the second dual of a Banach algebra, Bull. Austral. Math. Soc. 83 (2011), 222-129.

5. D. Benkovič, Biderivations of triangular algebras. Linear Algebra Appl. 431 (2009), 1587-1602.

6. M. BREŠAR, Commuting maps: A survey. Taiwanese J. Math. 8 (2004), 361-397.

7. H. G. Dales, F. Ghahramani and N. Grønbæk, Derivations into iterated duals of Banach algebras. Studia Math., 128 (1) (1998), 19-54.

8. Y. DU and Y. WANG, Biderivations of generalized matrix algebras. Linear Algebra. Appl. 438 (2013), 4483-4499.

9. B. E. Forrest and L. W. Marcoux, Derivations on triangular Banach algebras. Indiana Univ. Math. J., 45 (1996), 441-462.

10. B. E. Johnson, Weak amenability of group algebras. Bull. London Math. Soc. 23 (1991), 281-284.

11. V. Runde, Lectures On Amenability. Lecture Notes In Mathematics, 1774, Springer (2002).

12. Y. ZhAng, Weak amenability of module extension of Banach algebras. Trans. Amer. Math. Soc. 354 (2002), 4131-4151.

Sedigheh Barootkoob

Faculty of Basic Sciences

Department of Mathematics

P. O. Box 1339

University of Bojnord, Iran

s.barutkub@ub.ac.ir 